\title{
Virtual Machines Migration Technologies as a Strategy for Regional Manufacturing Companies
}

\author{
A.E. Bogomolov ${ }^{1}$, A.S. Kreymer ${ }^{1}$, and L.E. Popok ${ }^{1, *}$ \\ *Corresponding author: lpopok@gmail.com. \\ ${ }^{1}$ Kuban State Agrarian University, Krasnodar, Russia
}

\begin{abstract}
Modern approaches to the automation of business processes are increasingly confronted with questions of increase of efficiency of use of available computing resources. The transition to cloud computing technology makes the company to revise approaches to the assessment of their own capabilities and look for ways to reduce operating costs, it does not complicate the process of information infrastructure management. In the competitive environment of the organization following the path optimization of capital and operating costs through the introduction of new technologies, as well as through the transition and migration to a more cost-effective technologies.
\end{abstract}

Keywords: optimization, digital economy, digital technologies, migration, virtualization technologies, IT infrastructure.

\section{Introduction}

In modern conditions of large companies are increasingly using virtualization platform to reduce operating costs and simplify administration procedures, server systems and equipment as a set of computing resources and their logical combination abstracted from the hardware implementation. Using virtualization platform entails a corresponding additional costs for the purchase of software licenses virtualization platform. In such circumstances, it becomes important criterion TCO platform virtualization software licenses and lower costs for the purchase of these products.

The migration of virtual workloads is a strategically important step in the long-term cost savings associated with the purchase of software licenses for virtualization platform and provide competitive advantages for companies in various sectors in terms of economic instability and rising global competition.

In fact, the countries of the Eurasian Economic Union are in a need for services existing technological monopoly of large software vendors from the United States. In this context, questions to reduce costs for the use of distributed computing infrastructures are of particular relevance.

The relevance of this article due to the fact that most of the major regional companies are faced with the choice of cost-effective and reasonable choice of virtualization software platform manufacturers.

In the article the modern approaches to the implementation of the migration of virtual machines between different virtualization platforms. This article is the comparative characteristic instruments of migration of virtual workloads. The conclusions about the appropriateness of different approaches depending on the facing migration challenges and available resources. Author's technique of migrating virtual machines from VMware vSphere virtualization platform on the Microsoft Hyper- $\mathrm{V}$ virtualization platform, allowing to increase the speed and reliability of the migration process and to save on operating costs of the company.

The need for scientific research issues related to the migration of virtual workloads and systems based on Microsoft Hyper-V virtualization platform as the optimal virtualization platform, define the relevance of the topic and the research presented in this article.

Results that can be obtained through the migration of virtual machines, should be considered from the point of view of the transformation of business processes associated with service virtualization platforms and obtain economic benefits from the implementation of these processes. Obviously, the need to use both quantitative and qualitative indicators in determining the impact of migration on the activities of producer organizations.

\section{Methods}

The authors used such research methods as analysis, synthesis, description and comparison. Their use is determined by the nature of theoretical research, which included the following stages: formulation of the problem, analysis of information on the subject, the calculation of cost-effectiveness, comparison and description of the various migration tools.

As part of the testing tools are evaluated based on several criteria:

- delegation of rights and infrastructure;

- cost of a migration tool;

- $\quad$ support solution by vendor;

- the rate of migration / machine downtime; 
- $\quad$ requirements for disk resources for the migration;

- $\quad$ the automation of mass migration;

- rollback and recovery in case of problems.

\section{Findings}

\subsection{The economic rationale for migration of virtual workloads}

For the feasibility study of the migration virtual workloads with VMware vSphere virtualization platform on the Microsoft Hyper-V virtualization platform has been developed with inputs to the script, as well as an economic model for the calculation of the following indicators:

- $\quad$ Net Present Value (NPV);

- $\quad$ internal rate of return (IRR) for 5 years;

- payback initiatives;

- the cost of implementation of the initiative;

- $\quad$ savings from the implementation of the initiative.

The scenario describes the migration of virtual machines in 6000 for three years, a platform to host virtual machines are 200 physical servers. This scenario describes the migration of virtual machines with Windows operating system. All prices are in rubles, the model does not consider the situation of fluctuations in the value of licenses, depending on exchange rates.

Input data for economic models are presented in Table 1.

Table 1. Input data for an economic model for calculating expediency migration of virtual workloads.

\begin{tabular}{|l|c|c|}
\hline \multicolumn{1}{|c|}{ Parameter } & Value & $\begin{array}{c}\text { Unit of } \\
\text { measurement }\end{array}$ \\
\hline The number of servers (4 CPU x 16 Core) & 200 & psc \\
\hline The number of virtual machines (2 Core) & 6000 & psc \\
\hline Price VMware vSphere licenses 6 Enterprise Plus for 1 processor & 0,236 & million rubles. \\
\hline $\begin{array}{l}\text { Price Basic Support License / Subscription for VMware vSphere Enterprise Plus for } \\
61 \text { processor for 1 year }\end{array}$ & 0,070 & million rubles. \\
\hline $\begin{array}{l}\text { The price of Microsoft Windows Server 2019 license Datacenter Single OLP } \\
\text { 16License NL CoreLic }\end{array}$ & 0,482 & million rubles. \\
\hline Renewals Software Assurance Single SA OLP 16License NL CoreLic for 1 year & 0,160 & million rubles. \\
\hline The price of Microsoft Windows Server 2019 license Single CAL NL Device & 0,002 & million rubles. \\
\hline $\begin{array}{l}\text { The price of Microsoft Windows Server 2019 license Stnd Single OLP 2License NL } \\
\text { CoreLic }\end{array}$ & 0,007 & million rubles. \\
\hline The sales price of the migration project, per year & 12 & million rubles. \\
\hline Discount rate & 10 & $\%$ \\
\hline Inflation rate & 5 & $\%$ \\
\hline
\end{tabular}

Source: Authors.

Figure 1 provides a summary graph of the results of economic modeling initiatives migration virtual workloads from one virtualization platform to another. 


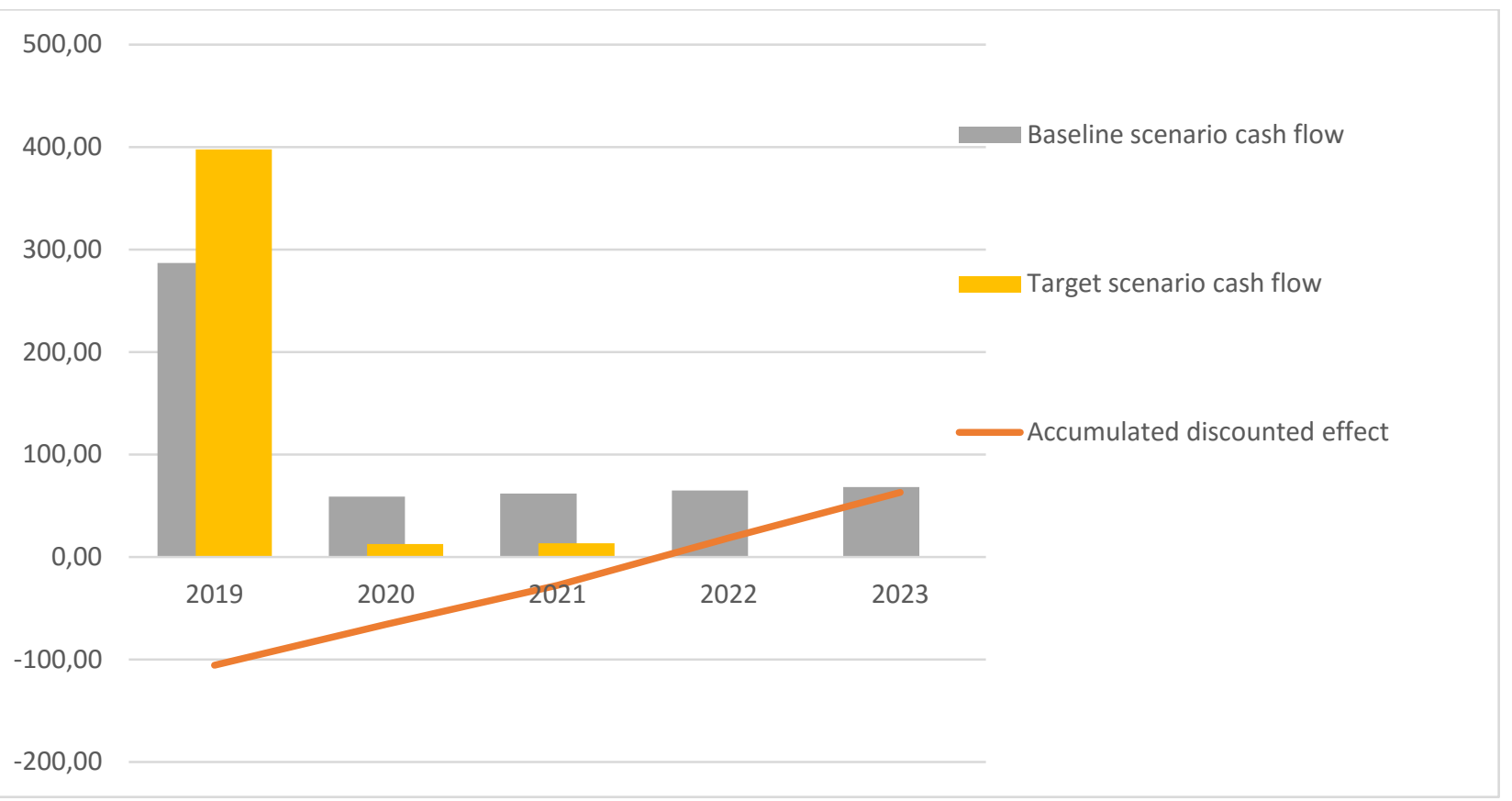

Fig. 1. The graph of the results of economic modeling (Source: Authors).

The graph shows the payback period comes less than three and a half years.

Major economic modeling results are presented in Table 2.

Table 2. Main results of economic modeling.

Source: Authors.

\begin{tabular}{|l|c|}
\hline \multicolumn{1}{|c|}{ Parameter } & Value \\
\hline Net Present Value (NPV), mln. Rub. (5 years) & 63 \\
\hline Internal rate of return (IRR),\% (over 5 years) & 33 \\
\hline The payback period for the discounted effect (DPP), years & 3.6 \\
\hline The costs for the implementation of the initiative, mln. Rub. (5 years) & 423 \\
\hline The savings from the implementation of the project, mln. Rub. (5 years) & 116 \\
\hline Payback period (PBP), years & 3.3 \\
\hline Net Present Value (NPV), mln. Rub. (5 years) & 63 \\
\hline
\end{tabular}

Based on the results obtained in economic modeling, we can talk about the feasibility and importance of launching initiatives on migration virtual workloads with VMware vSphere virtualization platform to platform Microsoft Hyper-V virtualization.

\subsection{Evaluation Criteria migration tools}

As part of the testing tools are evaluated based on several criteria:

- delegation of rights and infrastructure;

- $\quad$ cost migration tool;

- $\quad$ support solutions provider;

- the rate of migration / machine downtime;

- requirements for disk resources for the migration;

- the automation of migration;

- rollback and recovery in case of problems.

Below is the basic information about each instrument and score each item on a scale. It is further provided a brief summary table with the scores on the estimated parameters and offered the most effective options.

Microsoft System Center Virtual Machine Manager (SCVMM).SCVMM allows you to connect VMware vCenter management servers from a single console and see all the virtualization nodes and all virtual machines with the possibility of creating, managing and converting machines. In this test only considers aspects of the migration of virtual machines on Hyper-V.

The first step in considering the method of migration is the question of delegation of least privilege migration team for industrial VMware vSphere environment. According to the official Microsoft documentation to add vCenter in 
VMM requires an account with full rights to the entire infrastructure of VMware vSphere and local administrative rights on the vCenter. On a scale of SCVMM is estimated at 4 points on the criterion of the delegation and on the infrastructure of the rights due to the requirements of local recording on the nodes [1].

The second important point is to support the decision. SCVMM is part of System Center, the flagship product to Microsoft, periodically updated, supported by the software, and is estimated at 5 points on the criterion of support.

Another criterion is the speed of migration downtime virial machines for the period of migration. SCVMM migrates the virtual machine off, copying it to VMDK disk format to the HTTP protocol node and completing the conversion in VHDX after copying. unavailability of time for a typical stage of a virtual machine test was 1 hour 36 minutes from the start of the process before the end of migration and access to virtual machines over the network. Based on the criterion of the migration rate -2 points.

From the viewpoint of resource requirements, the destination node requires double the volume allocation of disk space used by the virtual machine: copying disk and its conversion. After conversion, the original disk copy is removed and used a copy of the virtual disk. Based on the criterion of resource requirements -2 points.

Process automation is one of the key factors for mass migration. SCVMM automatically selects the least loaded node that you are migrating to a virtual machine and a free disk to house its files. The virtual machine is highly available immediately connected to the virtual networks managed by the VMM, receives a specified IP address from a special pool. All operations can be done from the GUI once and save it as a PowerShell script for subsequent modifications. In this case, some of the complex migration scenarios, for example - the virtual machines with nonstandard settings or additional requirements, may require rework scripts. Or the development of new scripts, if it would be more appropriate. The object model and scripting SCVMM (PowerShell) language allows such improvements. Based on automation criteria - 5 points.

When a virtual machine migration tool SCVMM source machine on the VMware side is turned off and remains there at the end of the migration, requiring manual removal. This allows simple incorporation of the original virtual machine, as the abolition of migration plan. Based on the criterion of recovery - 5 points.

SCVMM is a paid product, licensed on processor virtualization host, but is included in the package, along with licenses for Windows Server, and, as a rule, is already available in most large organizations. Based on the criterion of cost - 3 points.

Microsoft Virtual Machine Converter (MVMC).MVMC is a separate Microsoft product for machine migration with VMware vSphere infrastructure (either physical servers) on the Hyper-V (or Microsoft Azure) [2].

In terms of delegating enough to MVMC minimum role configured for VMM migration tool. Rights vSphere nodes is required. Based on the criterion of delegation of rights infrastructure - 5 points.

At the current time MVMC is an unsupported product. Based on the criterion of support - 1 point.

During migration, shut down the machine is copied, then converted. It takes 32 minutes for a typical test virtual machine. Based on the criterion of the migration rate -3 points.

MVMC requires double the amount of disk space on the conversion time. Based on the criterion of resource requirements - 2 points.

Tasks performed by MVMC, partially automated. Connecting to the network, clustering of virtual machines, the installation of component integration, the selection addresses, all this can be done in PowerShell; but the choice of Hyper- $\mathrm{V}$ host and the drive to accommodate loads require explicit administrator's decision. Evaluation criteria for automation - 3 points.

When a virtual machine migration tools MVMC original machine on VMware side off and stays there for the end of the migration, requiring manual removal. That enables simple on both the original VM migration plan cancellation. Based on the criterion of recovery - 5 points.

MVMC is a free product and does not require a purchase. Based on the criterion of cost -5 points.

5nine Easy Converter.5nine Easy Converter (hereinafter 5nine) - this is a third-party solution V2V. It comes in the form of free and paid versions, where the paid version has the ability to automate. The advantage compared to the VMM / MVMC is to support the virtual machines of the second generation, in that it can convert the machine VMware vSphere, use the UEFI boot mode [3].

In terms of delegating side vCenter to 5nine lack minimum role that is configured to perform the migration in VMM. If you are migrating directly from VSphere require superuser (root) on the nodes. Based on the criterion of delegating infrastructure - 4 points.

5nine is a third-party product, the paid version is supported by the developer. Microsoft support incidents do not take to convert a third-party product, but to convert the machine is supported by Hyper-V. Based on the criterion of support - 3 points.

Speed machines and inaccessibility of the migration to 5nine comparable MVMC Migration with vCenter. Turning off the machine is copied, then converted. It takes 37 minutes for a typical test virtual machine vCenter. Based on the criterion of the migration rate -3 points.

5nine requires double the amount of disk space on the conversion time. Based on the criterion of resource requirements - 2 points

Tasks performed by the free version 5nine, not automated. For the paid version, there PowerShell module of four cmdlets to automate the migration directly from vSphere. This module does not support the migration from the vCenter, and since, as a rule companies have hundreds of vSphere hosts and virtual machines are automatically moved between 
them, it imposes restrictions - machines will need to pre-party VMware to migrate to a single node, which is offbalancing technology. Evaluation criteria for automation - 3 points.

When a virtual machine migration tools 5nine original machine on the side of VMware removed. To cancel a migration should first make a clone of the machine (can be automated, but requires a disk resource) or to restore the original virtual machine from a backup. Based on the criterion of recovery -3 points.

5nine Easy Converter in an embodiment of the automation solution is chargeable and licensed for each VM at prices comparable with Azure; 25-30 \$ per virtual machine. Based on the criterion of cost - 2 points.

Scripting solution based on NFS, VMotion and free utilities. As an alternative commercial solution offers an option that allows you to significantly reduce the downtime of virtual machines on the migration period. On a Windows server is one of the drives available in both shared with the SMB protocol (standard Windows shared folders), and the NFS protocol (a protocol for shared disk of UNIX, also supports VMware VSphere). On the side of the VMware vCenter NFS shared volume is added as a storage and virtual machine is transferred to it is turned on using vMotion technology. After the machine files will be on a Windows server, the machine is turned off, and her drive from VMDK format instantly converted first into the VHD, and then VHDX by changing the header files of the virtual disk utilities VHDtool and VHDXtool. Further PowerShell scripts created for the VMM target highly available VM on Hyper-V with the same parameters as the original, it is connected obtained VHDX disk. The virtual disk, even in the off state, the integration components are installed. The virtual machine is turned on, it takes a restart, the virtual machine gets old IPaddress and is ready for use after a few minutes of unavailability [4]. In terms of delegating side vCenter requires only two rights - Clone (create a copy for the abolition of migration) and moving disks on NFS. Rights to vSphere is not required. Based on the criterion of delegation of rights infrastructure -5 points.

The most difficult issue for the described solution is its support. Since conversion is not used for any of Microsoft products, problems with conversion formal support cannot receive. However, if at the end of the conversion of a virtual disk in VHDX format the disk has mounted, it has created a machine Hyper-V has standard support for Microsoft. Based on the criterion of support - 4 points.

The virtual machine is transferred to the Windows server is turned on without stopping services. Converting a virtual machine is performed for a few seconds. Downtime is equal to two times reboots the operating system. Based on the criterion of the migration rate - 5 points. Additional disk space on the process of conversion is not required. Based on the criterion of resource requirements - 5 points. The solution is fully built on scripts using PowerShell module for VMM and PowerCLI from the VMware, as well as third-party freeware utilities nfsutil, vhdtool and vhdxtool. All tasks can be automated [5]. Based on automation - 5 points. When a virtual machine migration tools Storage vMotion VMware source machine files are transferred on the NFS, where on the site will be converted to another format. Disk Copy does not occur at any of the stages, so copies of the original virtual machine remains. To cancel a migration should first make a backup of the virtual machine. Based on the criterion of recovery - 3 points. This approach does not use a fee-based products and does not require a purchase. Based on the cost -5 points. Test results. The results of the testing migration tools virtual workloads are presented in Table 3.

Table 3. Criteria for evaluating the migration tool virtual workloads

Source: Authors.

\begin{tabular}{|c|c|c|c|c|}
\hline Evaluation criteria & SCVMM & MVMC & 5nine & scripts \\
\hline Delegation and the right infrastructure & 4 & 5 & 4 & 5 \\
\hline Support Solutions & 5 & 1 & 3 & 3 \\
\hline Service downtime & 2 & 3 & 3 & 5 \\
\hline Resource Requirements & 2 & 2 & 2 & 5 \\
\hline Automation & 5 & 3 & 3 & 5 \\
\hline Rollback and recovery & 5 & 5 & 3 & 2 \\
\hline Cost & 3 & 5 & 2 & 5 \\
\hline TOTAL & 26 & 24 & 20 & 30 \\
\hline
\end{tabular}

From the resulting table, taking into account the points described by the criteria of evaluation of each approach shows that the best approach - migration via scripting method. You can also recommend the use of the migration method using SCVMM to migrate those virtual workloads that for some technical reasons, cannot be migrated using the scripting approach.

\section{Conclusion}

Migration of virtual machines with the VMware vSphere virtualization platform undoubtedly brings many economic benefits. However, this migration requires considerable efforts, the launch of a full-fledged design and selection of the correct migration tool to reduce the risks associated with an unplanned "downtime" for mission-critical applications. In addition, the personnel involved in the migration process should be highly qualified and well-known sequence of actions. 
It should be remembered that the implementation and debugging of VM migration procedures can be timeconsuming, which in the case of incorrect project planning may lead to a further increase in costs - the specific result depends on the choice of migration tools, the nature of the functions of personnel management and related project management.

Thus, there is a large enough reserve in reducing administrative cost of computing infrastructures. This is important in the formation of the integration processes between economic actors from EU countries and the maintenance of a single economic space.

\section{References}

1 Microsoft, Convert a VMware VM to Hyper-V in the VMM fabric (2019). URL: https://docs.microsoft.com/enus/system-center/vmm/vm-convert-vmware?view $=$ sc-vmm-2019. Accessed: 30.08 .2019 .

2 Microsoft, Microsoft Virtual Machine Converter 3.0 (2017). URL: https://docs.microsoft.com/en-us/previousversions/windows/it-pro/windows-server-2012-r2-and-2012/dn873998(v\%3Dws.11). Accessed: 30.08.2019.

3 5nine Software, Inc., User Guide .5nine V2V Easy Converter Version 7.0. Description PowerShell API and Microsoft Azure API (2016). URL: https://www.5nine.ru/Docs/5nine Easy V2V Converter_API v7.pdf. Accessed: 30.08.2019.

4 VMBlog, Converting VMware virtual machines to Hyper-V format (2017). URL: https://vmblog.ru/konvertaciyavirtualnoj-mashiny-vmware-v-format-hyper-v/. Accessed: 30.08.2019. [in Rus.].

5 Microsoft, Understanding a Windows PowerShell Module (2016). URL: https://docs.microsoft.com/enus/powershell/developer/module/understanding-a-windows-powershell-module. Accessed: 30.08.2019. 